\title{
Complete phase diagram for coherent vortex formation in a two-dimensional inviscid fluid in an annulus
}

\author{
Peilong Chen and M. C. Cross \\ Condensed Matter Physics 114-36, California Institute of Technology, Pasadena, California 91125
}

(Received 19 July 1996)

\begin{abstract}
By calculating the two-dimensional solutions and the second-order bifurcation analysis of the mean field equations of a two-dimensional ideal fluid, we are able to complete the phase diagram of coherent vortex formation in an annulus, which we have partially calculated in a previous paper [Phys. Rev. E 50, 2022 (1994)]. The complete phase diagram demonstrates the advantage of the statistical mechanics approach to determine the final equilibrium directly from the initial condition. [S1063-651X(97)00408-X]

PACS number(s): 47.20.-k, 05.20.Gg, 52.25.Kn, 92.90.+x
\end{abstract}

Recently statistical equilibrium of a single coherent vortex in a two-dimensional (2D) incompressible ideal fluid has been studied in a disk, using the point vortex model [1] and the continuous vorticity mean field theory [2]. Properties of the vortex depending on the system parameters are calculated and an interesting bifurcation from a symmetric vortex at the disk center to an off-center vortex was also found. In a previous paper [3] we have solved the mean field equations in an annulus which has an important additional parameter, namely, the constant inner circulation, providing a background shear in the system. Calculation in an annulus is also closer to the application of the theory to the coherent structures on planetary atmospheres. By calculating the symmetric solutions, the bifurcation point to single-vortex states, and the approximate single-vortex maximum energy, we estimated the parameters (total vorticity, energy, angular momentum, and inner circulation) of initial conditions leading to the formation of single coherent vortices. In this brief report, we explicitly solve for the $2 \mathrm{D}$ single-vortex solutions and also perform a second-order bifurcation analysis on the symmetric solutions. With these calculations we complete the phase diagram, especially determining the parameter range with first-order transitions, which was inaccessible to the previous methods, and demonstrate the potential of the statistical mechanics approach in predicting the final equilibrium directly from the initial condition. [4]

The two-level mean field equations which we solve are

$$
-\nabla^{2} \psi_{0}(\mathbf{r})=\omega_{0}(\mathbf{r})=\frac{q}{1+\exp \left[\beta\left(q \psi_{0}+\Omega r^{2}-\mu\right)\right]},
$$

with $\psi_{0}(\mathbf{r})$ the equilibrium stream function and $\omega_{0}(\mathbf{r})$ the vorticity field. $q$ is the vorticity level of the initial uniform distribution and chosen to be one. $(\mu, \beta, \Omega)$ are parameters to be determined by the total vorticity $Q$, (kinetic) energy $E$, and angular momentum $M$

$$
\begin{gathered}
Q=\int \omega_{0} d \mathbf{r}, \\
E=\frac{1}{2} \int \psi_{0} \omega_{0} d \mathbf{r}+\frac{1}{2} \oint_{\text {boundaries }} \psi_{0}\left(\nabla \times \psi_{0} \mathbf{z}\right) \cdot d \mathbf{l},
\end{gathered}
$$

In an annulus in which we solve these equations, the other important initial quantity is the conserved circulation $C$ at the inner boundary,

$$
C=\left.\frac{1}{2 \pi} \int \frac{\partial \psi}{\partial r}\right|_{\text {inner boundary }} d \theta
$$

which provides a background shear on the flow [3].

Equation (1) is solved by an iterative scheme: a guessed initial vorticity distribution $\omega_{t}(\mathbf{r})$ is used to solve the Poisson equation $\nabla^{2} \psi_{t}=-\omega_{t}$. Next the $\psi_{t}$ just calculated is substituted into the right hand side of Eq. (1) and a new $\omega_{t}$ can be calculated with each set of $(\mu, \beta, \Omega)$. A root-seeking algorithm is then used here to find the set of $(\mu, \beta, \Omega)$ giving an $\omega_{t}$ with required $Q, M$, and $E$. Now the iteration is repeated until a converged $\omega_{t}(\mathbf{r})$ is reached. We can also choose $\beta$ initially and find $(\mu, \Omega)$ for a prescribed $(Q, M)$. On the other hand, we can numerically integrate Eq. (1) to find the axisymmetric solutions, and a second-order bifurcation analysis can be performed, which is described in details in Ref. [2]. From the bifurcation analysis, we can quickly find the bifurcation point (from the first-order calculation) as well as the bifurcation behavior (from the second-order results).

For $Q=2, M=4$, and $C=1$ in an annulus with inner radius one and outer radius two, solutions at four different energies are shown in Fig. 1. In this case, there is a bifurcation from the symmetric to single-vortex state (see Fig. 1 in [3]). The first graph, Fig. 1(a), has an energy smaller than the bifurcation energy and shows a symmetric vortex ring. As the energy is increased through the bifurcation energy in the other three graphs, the vortex ring gradually breaks up and finally reaches a single-vortex state at high energy. When these asymmetric solutions are plotted in an entropy-energy plot (Fig. 1 in [3]), they show a higher entropy than the symmetric states at the same energy, indicating thermodynamically more probable states.

The maximum energy state (corresponding to $\beta \rightarrow-\infty$ ) is a uniform vorticity patch with its shape strongly depending 


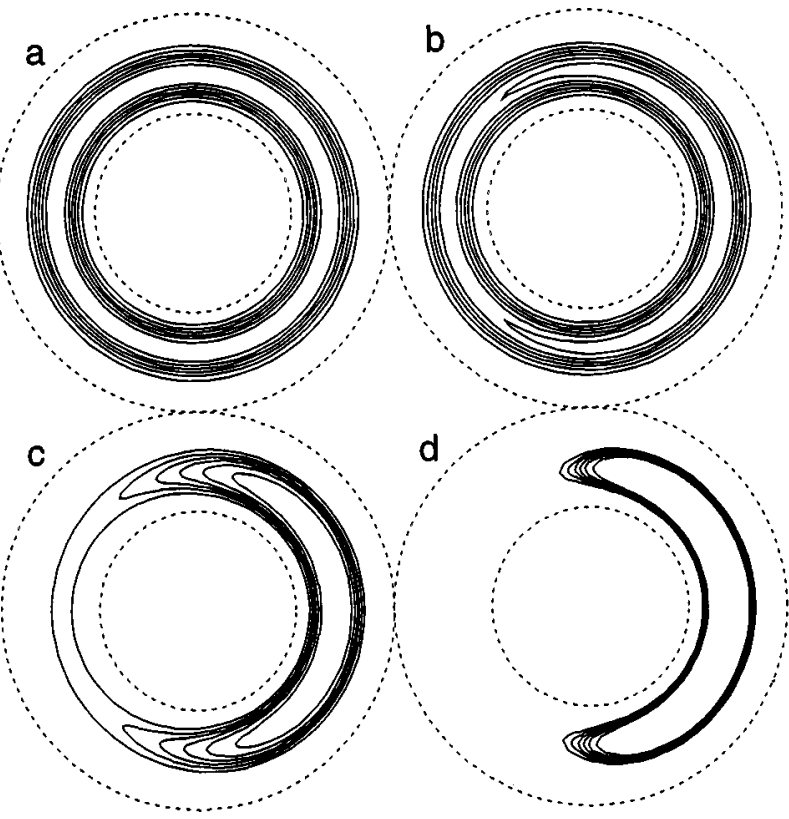

FIG. 1. Vorticity contours for $C=1, Q=2$, and $M=4$ in an annulus with inner radius one and outer radius two. (a) Symmetric solution at $E=1.5598$. (b) Asymmetric solution at $E=1.5622$. (c) $E=1.5773$. (d) $E=1.5939$. The bifurcation energy is 1.5619 . Contour levels are equally spaced.

on the inner circulation $C$ : as the circulation, and hence the positive background shear [3], increases, the shape becomes more elongated in the azimuthal direction and thinner in the radial direction. This is similar to the increase of the aspect ratio of an elliptical vortex with increasing shear flow found in analytic solutions [5].

To quantify the asymmetric states we define the average azimuthal angle spanned by a state, $s$, as

$$
s \equiv 4 \int_{0}^{\pi} \int_{a}^{b} \theta \omega(\mathbf{r}) r d r d \theta \int_{0}^{\pi} \int_{a}^{b} \omega(\mathbf{r}) r d r d \theta
$$

for a distribution centered at $\theta=0$, with $a$ and $b$ the inner and outer radii. The difference $\bar{s}$ between $2 \pi$ and $s$ can then be treated as the order parameter of the transition and it is zero for a symmetric state. If the bifurcation is a forward pitchfork bifurcation, $\bar{s}$ will scale as the square root of $\Delta E \equiv E-E_{\mathrm{c}}$, with $E_{\mathrm{c}}$ the bifurcation energy. For $C=1, \bar{s}$ plotted as a function of energy in Fig. 2 (the + 's) clearly shows this scaling behavior.

This scaling becomes apparent in the second-order bifurcation analysis [2] because $\bar{s}$ is then proportional to the perturbation and $\Delta E$ to the square of the perturbation. The results calculated from the analysis are shown as the solid lines in Fig. 2. For $C=1$ the solid line agrees perfectly with the single-vortex states (the + 's) at small $\bar{s}$ and the agreement remains quite good even when $\bar{s}$ approaches $\pi$. The positive value of $\Delta E$ found in the analysis for $C=1$ again confirms a forward pitchfork bifurcation.

However, as $C$ is lowered, $\Delta E$ from the bifurcation analysis becomes negative at a particular $C$, signifying a backward pitchfork bifurcation. Particular examples are shown in Fig. 2 for $C$ equal to -0.249 and -0.27 . In these situations the
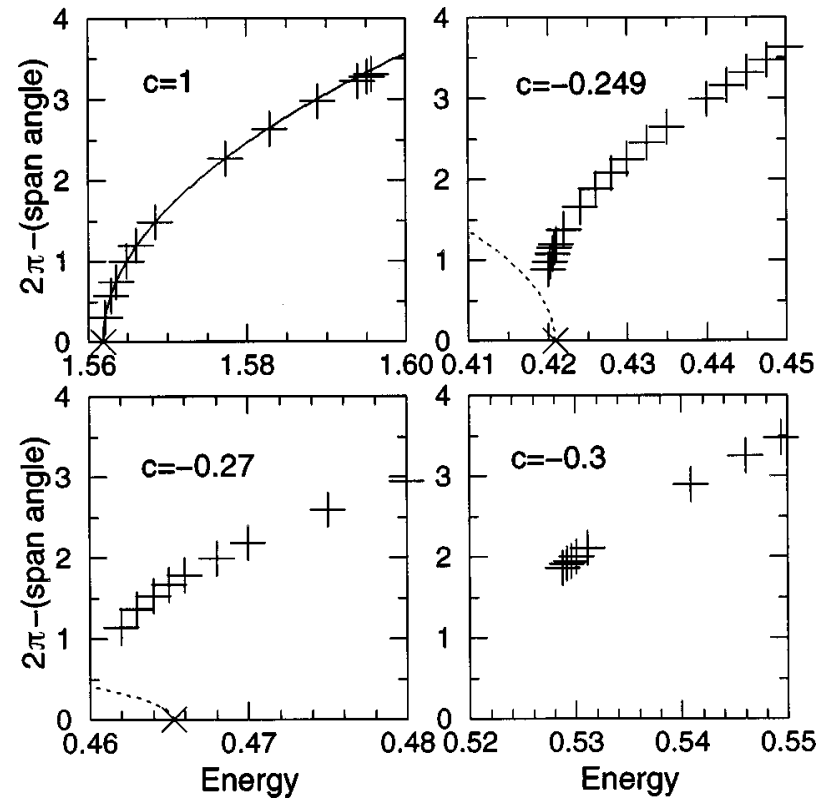

FIG. 2. $\bar{s}$ dependence on $E . \times$ indicates the bifurcation energy and + the asymmetric solution. Note that the symmetric solutions always have $\bar{s}$ equal to zero. The lines are the prediction from the second-order bifurcation analysis.

dotted lines trace from the bifurcation point (the $\times$ ) to the left and give unstable solutions close to the bifurcation point. Higher order terms in the expansion would bend the curve around to the right, presumably to merge with the stable solutions found numerically (the + 's). (Note that the fixedenergy numerical scheme does not find the unstable solutions as the iteration procedure always favors the stable solution at

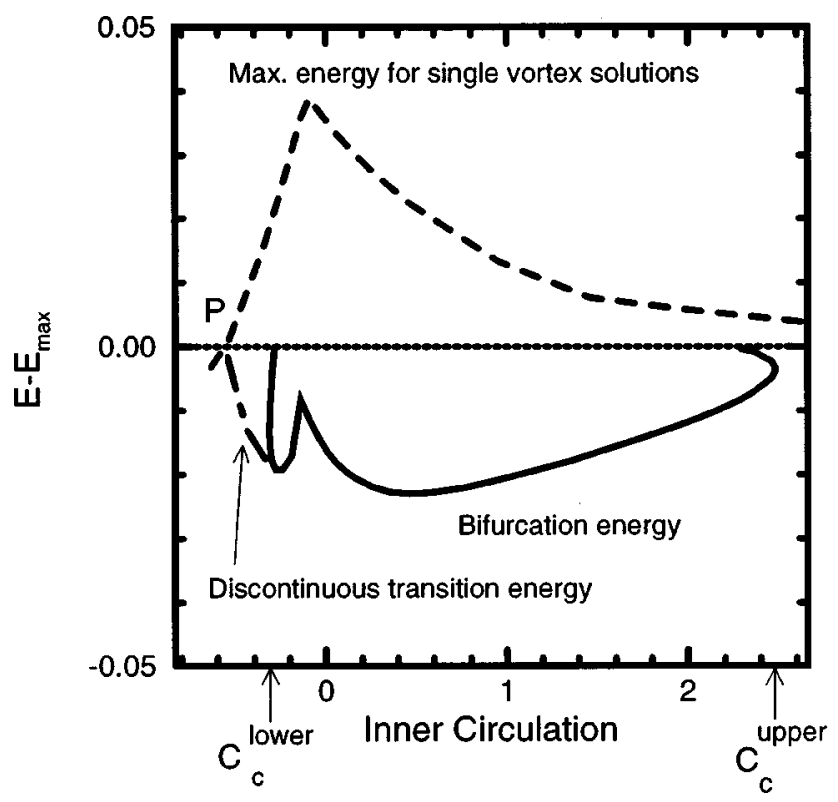

FIG. 3. Complete phase diagram for $Q=2$ and $M=4$. $E_{\max }$ represents the maximum energy of the symmetric solution. The solid line is the bifurcation line. The dotted and dashed lines are the maximum energy for the symmetric and asymmetric solutions, respectively. The dotted-dashed line is the first-order transition when a bifurcation is not present, i.e., where the entropies of the two solutions cross. 
that energy.) Thus a continuous transition from a symmetric state to a single coherent vortex exists for $C$ larger than a particular value, which is about -0.2 for $Q=2$ and $M=4$. Below this value the transition will be discontinuous.

For $C$ lower than $C_{c}^{\text {lower }}$ there is no linear instability of the symmetric state up to its maximum energy. Even without a bifurcation point, however, the single-vortex states still exist, as shown in Fig. 2 for $C=-0.3$. Nevertheless, as $C$ is further decreased, eventually a point is reached where the entropy of the asymmetric state becomes smaller than that of the symmetric state at the same energy. Hence the coherent single vortex is no longer a thermodynamically more probable state than the symmetric solution. One way to quickly determine the point is to compare the maximum energy of these two states. For $Q=2$ and $M=4$ this happens at about $C=-0.52$.

The complete phase diagram for $Q=2$ and $M=4$ is plotted in Fig. 3. The dashed and dotted lines represent the maximum energy for single vortex and symmetric solutions, respectively. Between $C_{c}^{\text {upper }}$ and $C_{c}^{\text {lower }}$ there is bifurcation indicated by the solid line. Below $C_{c}^{\text {lower }}$, the dashed-dotted line shows the energy above which the single-vortex state yields a higher entropy at that particular $C$, and point $P$ marks the crossing of the two maximum energy lines. With this phase diagram, we can quickly predict the final equilibrium for any initial flow. For inner circulation $C$ larger than the small negative value at $P$, we can have the formation of a stable coherent vortex. Using the relation between $C$ and the background shear $\sigma$ [3],

$$
\sigma \equiv r \frac{\partial}{\partial r}\left(\frac{u_{\theta}}{r}\right)=\frac{2 C}{r^{2}},
$$

we find that the critical ratio between the shear strength and vorticity level is about -0.5 . This result is consistent with the numerical simulations [6], where it is shown dynamically that a shear larger than a small negative value (ranging from -0.1 to -0.2 ) is needed to support a stable coherent vortex. As noted in the previous paper [3], if the conditions closer to those in the simulations are used in our calculations, smaller values of the ratio close to the simulation values are obtained.

The authors acknowledge the support of the National Science Foundation under Grant No. DMR 9311444.
[1] R.A. Smith and T.M. O’Neil, Phys. Fluids B 2, 2961 (1990); T.M. O'Neil and R.A. Smith, ibid. 4, 2720 (1992)

[2] P. Chen and M.C. Cross, Phys. Rev. E 54, 6356 (1996)

[3] P. Chen and M.C. Cross, Phys. Rev. E 50, 2022 (1994)

[4] J. Miller, Phys. Rev. Lett 65, 2137 (1990)J. Miller, P.B. Weichman, and M.C. Cross, Phys. Rev. A 45, 2328 (1992); R.
Robert and J. Sommeria, Fluid Mech. 229, 291 (1991)

[5] D.W. Moore and P.G. Saffman in Aircraft Wake Turbulence, edited by J. H. Olsen, A. Goldburg, and M. Rogers (Plenum, New York, 1971), p. 339.

[6] P.S. Marcus, J. Fluid Mech. 215, 393 (1990) 